\title{
Basal cerebral venous drainage from cavernous sinus dural arteriovenous fistulas
}

\author{
Hiro Kiyosue • Hiromu Mori • Yoshiko Sagara • \\ Yuzo Hori • Mika Okahara • Hirofumi Nagatomi • \\ Toshi Abe
}

Received: 8 June 2008 / Accepted: 8 December 2008 / Published online: 23 December 2008

(C) The Author(s) 2008. This article is published with open access at Springerlink.com

\begin{abstract}
Introduction This study aimed to define the patterns of basal cerebral venous drainage (BCVD) from cavernous sinus dural arteriovenous fistulas (CSDAVFs).

Materials and methods Forty sets of selective angiographic data from 36 patients with spontaneous CSDAVFs (age range, 53-79 years) were retrospectively analyzed for their drainage patterns. Three types of BCVD were observed, i.e., superolateral type, BCVD via the deep middle cerebral vein or uncal vein; posterolateral type, BCVD via the superior petrosal sinus and petrosal vein; and posteromedial type, $\mathrm{BCVD}$ via the bridging vein and the anterior pontomesen-
\end{abstract}

\footnotetext{
H. Kiyosue $(\bowtie) \cdot$ H. Mori $\cdot$ Y. Sagara

Department of Radiology, Oita University Faculty of Medicine,

1-1 Idaigaoka, Hasama,

Yufu City, Oita, Japan 879-5503

e-mail: hkiyosue@med.oita-u.ac.jp

Y. Hori

Department of Radiology, Nagatomi Neurosurgical Hospital,

Oita, Japan

M. Okahara

Department of Radiology, Oita University Faculty of Medicine, Oita, Japan

H. Nagatomi

Department of Neurosurgery, Nagatomi Neurosurgical Hospital, Oita, Japan

T. Abe

Department of Radiology,

Kurume University School of Medicine,

Kurume City, Fukuoka, Japan

M. Okahara

Department of Radiology, Shinbeppu Hospital,

Beppu, Japan
}

cephalic vein. MR images and/or 3D-DSA images were also reviewed when available.

Results BCVD from CSDAVF was found in 12 patients $(30 \%)$, and the other drainage routes included the superior ophthalmic vein in $25(63 \%)$, the inferior petrosal sinus in $17(43 \%)$, the superficial middle cerebral vein in $17(43 \%)$, intercavernous sinus in $15(38 \%)$, the superior petrosal sinus in seven (18\%), and pterygoid plexus in two (5\%), respectively. In 12 patients with BCVD, superolateral type was found in four (33\%), posterolateral type in five (42\%), and posteromedial type in seven (58\%). Four cases of posteromedial type were associated with other types of BCVD.

Conclusion CSDAVFs are often associated with BCVD via three different pathways. The posteromedial type via the bridging vein is the most frequent type of BCVD.

Keywords Cerebral angiography .

Dural arteriovenous fistula $\cdot$ Cerebral vein $\cdot$ Embolization

\section{Introduction}

Cavernous sinus dural arteriovenous fistulas (CSDAVFs) are generally considered as a benign type of intracranial DAVFs because the cavernous sinus (CS) has large and multiple extradural drainage routes including the inferior petrosal sinus, superior ophthalmic vein, superior petrosal sinus, pterygoid plexus, and intercavernous sinus [1]. However, aggressive symptoms such as brain edema, venous infarction, and/or hemorrhage can occur associated with dangerous venous drainages of retrograde cortical venous drainage via the superficial middle cerebral vein (SMCV) and basal cerebral vein (basal vein of Rosenthal) [2-7]. Among these dangerous venous drainages, the 
superficial drainage route of the SMCV has been well recognized $[8,9]$. However, basal cerebral venous drainage into the basal cerebral vein from CSDAVFs has not been documented in detail. In this study, we retrospectively analyzed the frequencies and patterns of basal cerebral venous drainage routes in CSDAVFs.

\section{Patients and methods}

We retrospectively analyzed 36 consecutive patients with spontaneous CSDAVFs who underwent cerebral angiography between August 1999 and July 2007, and reviewed their imaging findings. Patient age ranged from 53 to 79 years (mean age, 61 years), and there were six males and 30 females. Selective digital subtraction angiography of the internal and external carotid arteries and the vertebral artery were performed in all patients using biplane angiography equipments (Advantx LCN plus, GE Medical Systems, Milwaukee; Infinix VB, Toshiba Medical, Tokyo). Threedimensional reconstructed images of rotational angiography (3D-DSA) were obtained in 15 patients. Each patient gave written informed consent before examinations. Among 36 patients, 26 patients were treated by transvenous embolization and three patients by stereotactic radiosurgery, and the remaining seven patients were observed without treatment.

Forty sets of selective cerebral angiographic data including bilateral external and internal carotid angiography and ipsilateral vertebral angiography before treatment were reviewed by two experienced neuroradiologists, with consensus. In four of the seven patients untreated, followup angiography showed significant changes of the drainage pattern of CSDAVF were also reviewed. MR angiography, post-contrast fat-suppressed 3D gradient echo MR images, 3D-DSA images, and selective venography of the cavernous sinus during transvenous embolization were also reviewed when available.

Basal cerebral venous drainage routes were classified into three types based on cerebral angiographic findings and additional imaging findings (Fig. 1). Superolateral drainage involved the DAVF draining from the CS superolaterally via the superficial middle cerebral vein and into the deep middle cerebral vein or the uncal vein connecting to the basal cerebral vein (Figs. 2 and $4 \mathrm{c}$ ). Lateral drainage was characterized by the DAVF draining posterolaterally via the superior petrosal sinus and petrosal vein into the lateral mesencephalic vein into the basal cerebral vein (Fig. 3). Finally, posteromedial drainage involved the DAVF draining from the cavernous sinus posteromedially via the prepontine bridging vein to the anterior pontomesencephalic vein connecting to the basal cerebral vein (Fig. 4).

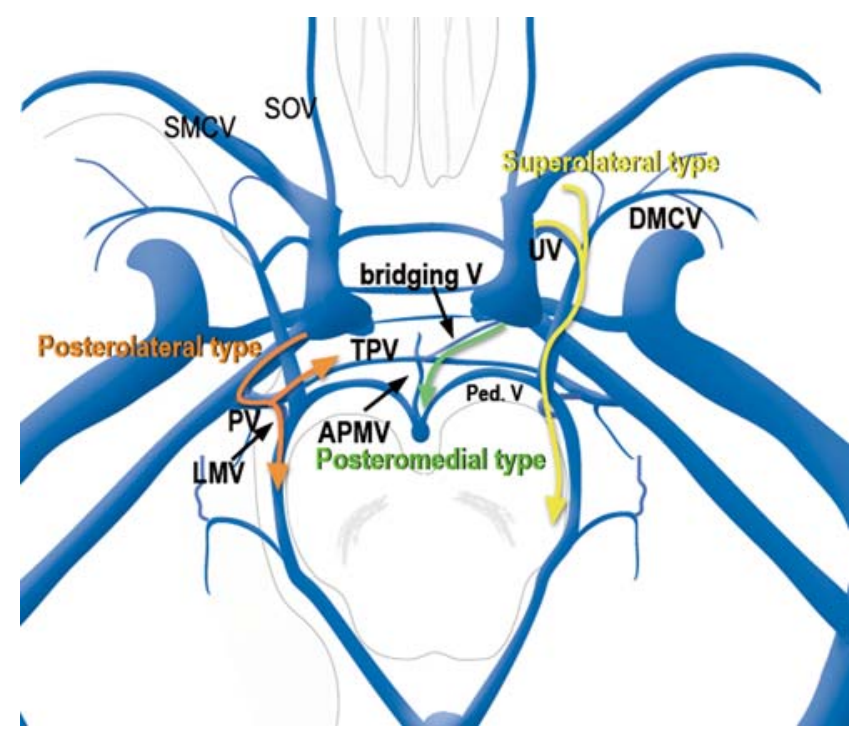

Fig. 1 Schematic drawing of the basal cerebral venous drainage routes. Superolateral type (yellow arrow): draining routes from cavernous sinus via the superficial middle cerebral vein or the uncal vein (UV) and/or the deep middle cerebral vein (DMCV) connecting to the basal cerebral vein. Posterolateral type (orange arrows): draining routes from cavernous sinus via the superior petrosal sinus and petrosal vein $(\mathrm{PV})$ connecting to the lateral mesencephalic vein (LMV) and/or the transverse pontine vein (TPV) into the basal cerebral vein. Posteromedial type (green arrow): draining routes from cavernous sinus via the prepontine bridging vein to the transverse pontine vein and/or the anterior pontomesencephalic vein (APMV) connecting to the basal cerebral vein. Ped.V peduncular vein, $S M C V$ superficial middle cerebral vein, $S O V$ superior ophthalmic vein

\section{Results}

All drainage routes analyzed from the 40 sets of angiography in the 36 patients are summarized in Table 1. Basal cerebral venous drainage from CSDAVFs was observed in 12 patients (30\%). Basal cerebral venous drainage was also associated with other drainage routes and was more frequently observed in cases with occlusion of the inferior petrosal sinus. There were ten of $24(42 \%)$ cases with occlusion of the inferior petrosal sinus versus two of $16(25 \%)$ cases with patent inferior petrosal sinus. Additional or alternative drainage routes included the superior ophthalmic vein in $25(63 \%)$, inferior petrosal sinus in $17(43 \%)$, superficial middle cerebral vein in $16(40 \%)$, intercavernous sinus in 15 (38\%), superior petrosal sinus in seven (18\%), and pterygoid plexus in two (5\%) patients.

Among the basal cerebral venous drainage cases, the superolateral type was found in four (33\%), posterolateral type in five (42\%), and posteromedial type in seven (58\%) patients (Table 2). Four cases of the posteromedial type were associated with other types of basal cerebral venous drainage (superolateral type in three and posterolateral type in one; Fig. 4). One patient with superolateral type of basal cerebral venous drainage showed hemorrhagic infarction of the deep 
Fig. 2 CSDAVF with superolateral type of basal cerebral venous drainage. a, b Frontal (a) and lateral (b) views of right external carotid angiogram show the AVF draining into the basal cerebral vein (arrowheads) via the uncal vein and the deep middle cerebral vein (arrows). Additional drainage routes of the superior ophthalmic vein, the inferior petrosal sinus, intercavernous sinus, and the temporal cortical veins are also noted
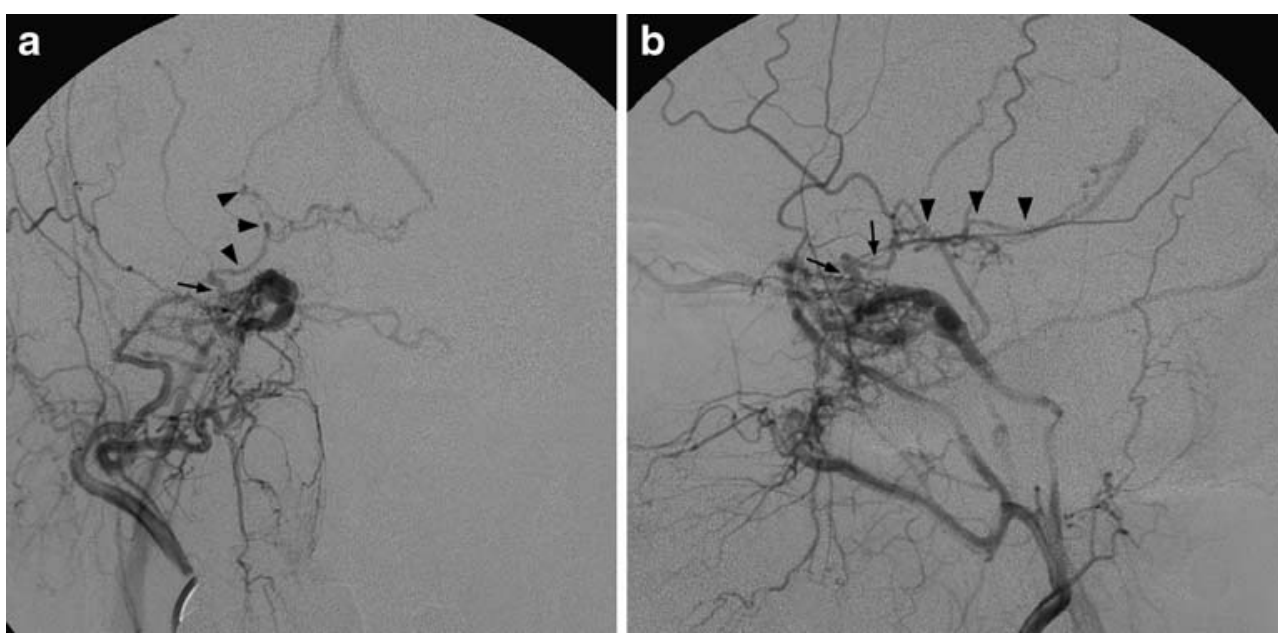

temporal lobe before treatment, and another patient with posteromedial type of basal cerebral venous drainage developed transient brain stem edema after incomplete transvenous embolization. No other cases showed cerebral symptoms.

\section{Discussion}

Symptoms of DAVFs are related to the types of venous drainage [1]. Retrograde cortical venous drainage through the SMCV and basal cerebral venous drainage into the basal cerebral vein and/or veins of the posterior fossa have potential risks of aggressive symptoms [2-7]. In basal cerebral venous drainages, hemorrhage and/or venous infarction can occur in deep areas of the brain, e.g., brain stem hemorrhage can cause fatal damage. In a recent study of 58 patients with CSDAVF, cerebral symptoms of hemorrhage, infarction, or venous congestion in the basal ganglia, brain stem, or cerebellum were found in $5 \%$ of patients and were significantly associated with cortical and
Fig. 3 CSDAVF with posterolateral type of basal cerebral venous drainage. a, b Frontal (a) and lateral (b) views of the left internal carotid angiography show the AVF draining posteriorly via the superior petrosal sinus (SPS) and the petrosal vein into the basal cerebral vein (arrows) and the cerebellar veins. Occlusion of the inferior petrosal sinus and anterior drainage via the superior ophthalmic vein are also seen. c Lateral view of maximum intensity projection image of 3D-DSA demonstrates the posterolateral type of basal cerebral venous drainages via the superior petrosal sinus (SPS) and petrosal vein $(\mathrm{PV})$ into the transversepontine vein(TPV)the anterior pontomesensephalic vein (APMV) and the lateral pontomesencephalic vein (LPMV) connecting to the basal cerebral vein $(\mathrm{BV})$. $S O V$ superior ophthalmic vein
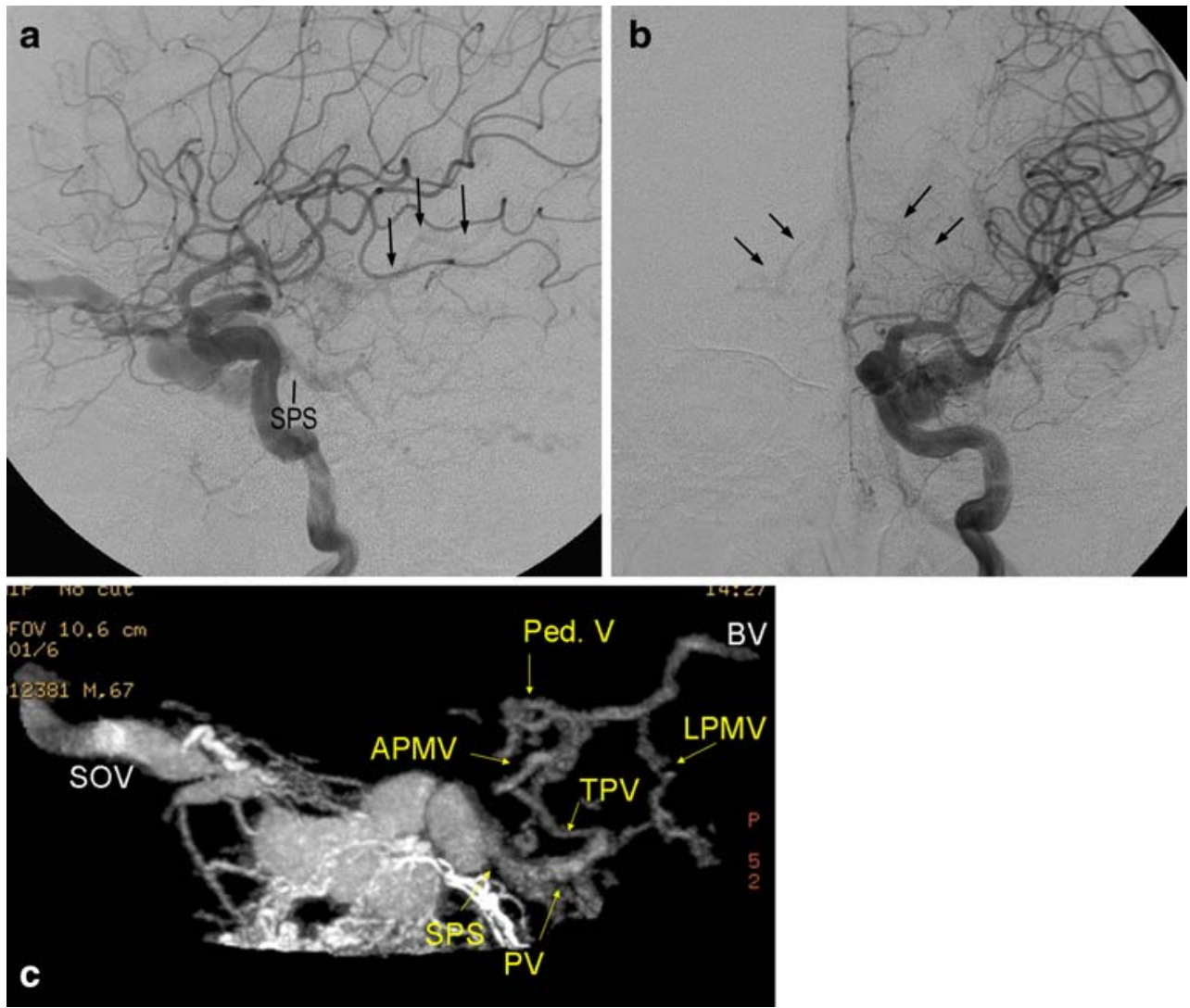

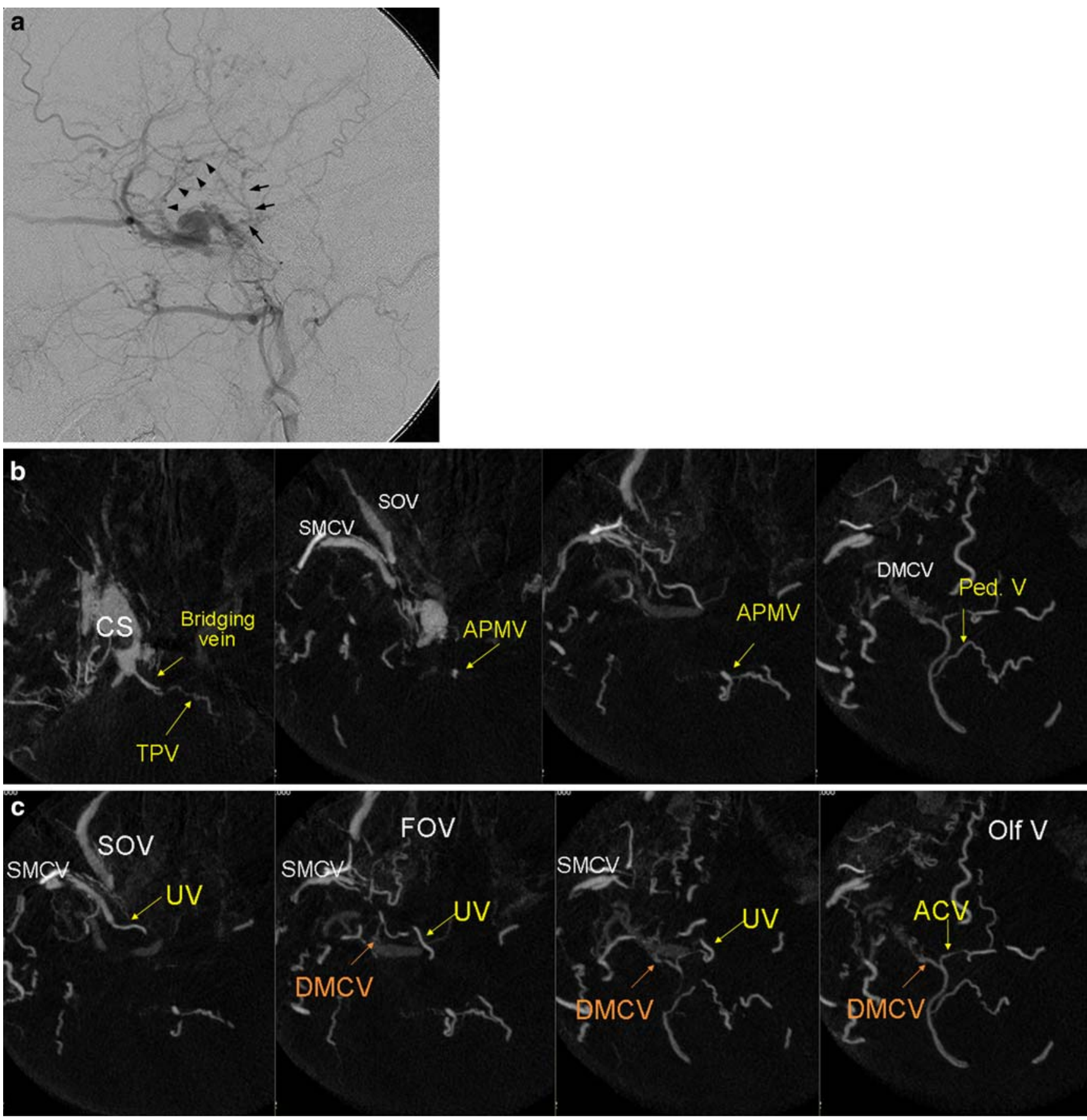

Fig. 4 CSDAVF with posteromedial type and superolateral type of basal cerebral venous drainage. a Lateral views of right external carotid angiogram shows the CSDAVF draining into the basal cerebral vein via the superorateral type of drainage route (arrowheads) and posteromedial type of drainage (arrows). b Axial reformatted images of the 3D-DSA from the level of cavernous sinus to the cerebral peduncle demonstrate the posteromedial type of basal cerebral venous drainage. The CSDAVF drains via the bridging vein into the transverse pontine vein (TPV), the anterior pontomesencephalic vein (APMV), and peduncular vein which connects to the basal cerebral vein. $C S$ cavernous sinus, $S M C V$ superficial middle cerebral vein, $D M C V$ deep middle cerebral vein. c Axial reformatted images of the 3D-DSA from the level of superior ophthalmic vein (SOV) to the cerebral peduncle demonstrate the superolateral type of basal cerebral venous drainage. The CSDAVF drains via the uncal vein (UV) and the deep middle cerebral vein (DMCV) arising from the superficial middle cerebral vein (SMCV) into the basal cerebral vein. Additional drainage routes of frontoorbital vein (FOV) and olfactory vein (Olf V) into the frontal base are also noted. $A C V$ the anterior cerebral vein communicating between the uncal vein and the basal cerebral vein 
Table 1 Drainage routes from CSDAVFs.

Drainage Routes No of Cases

IPS

IPS, interCS

1

IPS, BCV*

IPS, interCS, Pplx

IPS, interCS, SPS

IPS, SOV, SPS, InterCS

IPS, SOV, SPS, Pplx, InterCS

IPS, SOV

SOV

SOV, interCS

SOV, BCV*

SOV, SMCV

SOV, SMCV , BCV*

SOV, SMCV, SPS

SOV, SMCV, InterCS

SOV, SMCV , IPS, BCV*

SOV, SMCV , interCS , BCV*

SMCV

SMCV, SPS

SMCV, BCV*

SMCV, IPS, SPS, interCS

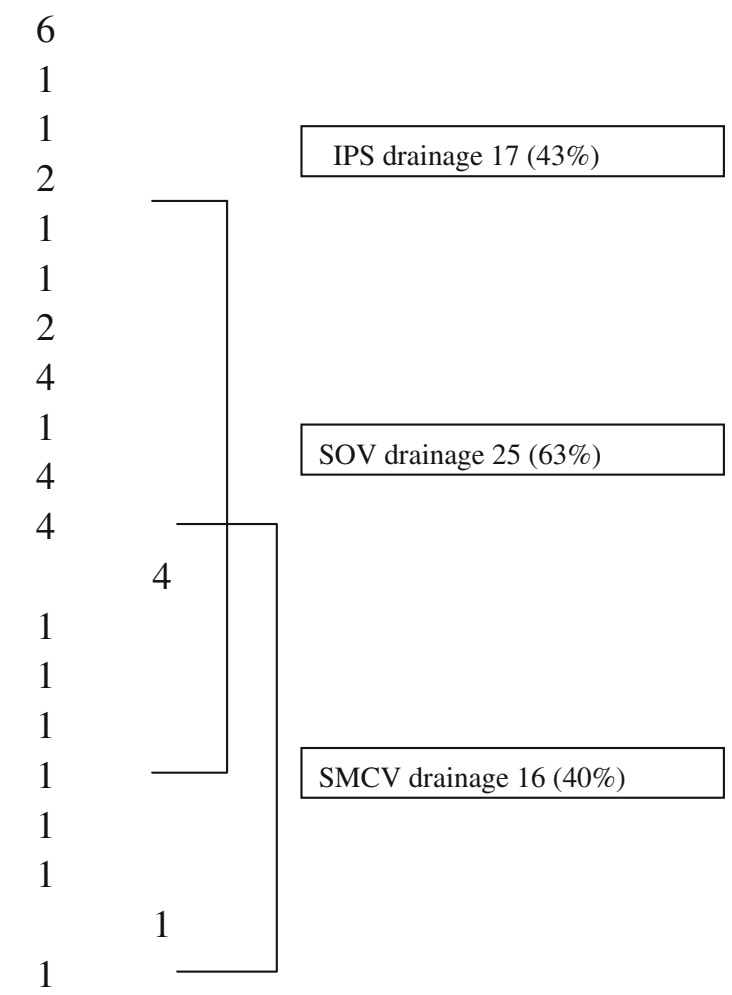

*BCV drainage $12(30 \%)$

IPS: inferior petrosal sinus, interCS: intercavernous sinus, Pplx: pterygoid plexus, SPS: superior petrosal sinus, SOV: superior ophthalmic vein, BCV:

basal cerebral vein, SMCV: superficial middle cerebral vein

$I P S$ inferior petrosal sinus, interCS intercavernous sinus, $P p l x$ pterygoid plexus, SPS superior petrosal sinus, $S O V$ superior ophthalmic vein, $B C V$ basal cerebral vein, $S M C V$ superficial middle cerebral vein

Table 2 Types of basal cerebral venous drainage from CSDAVFs.
Drainage Types

No of Cases $(\%)$

Superolateral type

Superolateral \& Posteromedial type

Posteromedial type

Posteromedial type \& Posterolateral type

Posterolateral type

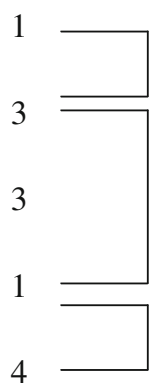


basal cerebral venous drainages [6]. Anatomically, the CS potentially communicates with the basal cerebral vein via several venous anastomoses [10]. In the embryonic periods, the cortical and pial veins developing into the SMCV are connected with the transverse sinus through a primitive tentorial sinus. The SMCV anastomoses with the cavernous sinus at the anterolateral aspect after developing to the prenatal stage or after birth [11-13]. The tentorial sinus regresses, and the cavernous sinus becomes the main drainage sinus from the SMCV in adults. During the development of the CS and SMCV, the deep pial veins of the deep middle cerebral vein and uncal veins communicate with the SMCV or CS at the anterolateral aspect of the CS. The basilar vein develops in the secondary longitudinal anastomoses between several separate components of the deep pial veins such as the deep telencephalic vein which forms the deep middle cerebral vein and the anterior cerebral vein. Therefore, the basal cerebral vein can involve pial anastomoses with superficial cerebral veins in both the cerebral and cerebellar fossae via the deep middle cerebral vein, uncal vein, anterior pontomesencephalic vein, and lateral mesencephalic vein. These anastomoses may provide pathways from the CSDAVFs. In this study, basal cerebral venous drainage could be classified into three types, i.e., the superolateral, posterolateral, and posteromedial types. The superolateral drainage type involves draining routes from the cavernous sinus through the SMCV and deep middle cerebral vein and/or uncal vein to the basal cerebral vein, and was found in $10 \%$ of CSDAVFs. A study involving the surgical observation of the SMCV demonstrated communication between the SMCV and the deep middle cerebral vein in 39 (45\%) of 82 hemispheres [14]. Although the superolateral type is relatively well known as a typical basal cerebral venous drainage, it was less frequently observed than the other types, in our present study. The posterolateral type involves draining routes from the cavernous sinus through the superior petrosal sinus, petrosal vein and lateral mesencephalic vein and/or anterior pontomesencephalic vein to the basal cerebral vein, and may also drain into the cerebellar veins. Some authors mentioned that this type of deep drainage results in potential risks of cerebral symptoms $[6,15]$. The posteromedial type is characterized by a draining route from the cavernous sinus via the prepontine bridging vein, transverse pontine vein, and/or anterior pontomesencephalic vein into the basal cerebral vein. Although only few case reports have described the posteromedial type of venous drainage via the prepontine bridging vein, it was the most frequently (18\% of all CSDAVFs) observed in our study [3]. Matsushima et al. reported that the bridging veins run from the anterior pontomesencephalic vein or transverse pontine vein to the posterior portion of the cavernous sinus just below the Meckel's cave [16]. They showed that bridging veins to the cavernous sinus were identified in $12.5 \%$ of 25 adult cadavers.

For the treatment of CSDAVFs by transvenous embolization, it is important to recognize that the three types of basal cerebral venous drainage routes originate from different portions of the cavernous sinus of the superolateral part including the SMCV, posterolateral part of the superior petrosal sinus, and posteromedial part adjacent to the inferior petrosal sinus. The outlet of these basal cerebral venous drainages should be infallibly occluded during transvenous embolization. Residual DAVFs with one type of these basal cerebral venous drainages have a high risk of cerebral or brain stem edema or hemorrhage when transvenous embolization results in incomplete occlusion of CSDAVF with residual basal cerebral venous drainage. Kim et al. reported transvenous embolization of CSDAVFs in 57 patients and showed that venous congestion of the posterior fossa after transvenous embolization developed in two patients [17]. The basal cerebral venous drainage routes can be overlooked in most cases of CSDAVF with multiple venous drainages because these veins are often tiny and can be superimposed by cerebral arteries and other drainage veins. Therefore, basal cerebral venous drainage types should be carefully evaluated before and during transvenous embolization of CSDAVFs. 3D-DSA would be helpful in evaluating the basal cerebral venous drainage in detail.

In conclusion, basal cerebral venous drainage is frequently associated with CSDAVFs which can drain via three different pathways. The careful assessment of drainage routes including basal cerebral venous drainage is required for successful treatment of CSDAVFs.

Conflict of interest statement We declare that we have no conflict of interest.

Open Access This article is distributed under the terms of the Creative Commons Attribution Noncommercial License which permits any noncommercial use, distribution, and reproduction in any medium, provided the original author(s) and source are credited.

\section{References}

1. Cognard C, Gobin YP, Pierot L et al (1995) Cerebral dural arteriovenous fistulas: clinical and angiographic correlation with a revised classification of venous drainage. Radiology 194:671-680

2. Satoh K, Satomi J, Nakajima N, Matsubara S, Nagahiro S (2001) Cerebellar hemorrhage caused by dural arteriovenous fistula: a review of five cases. J Neurosurg 94:422-426

3. Takahashi S, Tomura N, Watarai J, Mizoi K, Manabe H (1999) Dural arteriovenous fistula of the cavernous sinus with venous congestion of the brain stem: report of two cases. AJNR Am J Neuroradiol 20:886-888 
4. Phadke RV, Parihar A, Behari S, Sharma K (2006) Localized congestive venous encephalopathy associated with cavernous dural arteriovenous malformation. AJNR Am J Neuroradiol 27:1315-1317

5. Iwasaki M, Murakami K, Tomita T, Numagami Y, Nishijima M (2006) Cavernous sinus dural arteriovenous fistula complicated by pontine venous congestion. A case report. Surg Neurol 65:516519. doi:10.1016/j.surneu.2005.06.044

6. Suh DC, Lee JH, Kim SJ et al (2005) New concept in cavernous sinus dural arteriovenous fistula: correlation with presenting symptom and venous drainage patterns. Stroke 36:1134-1139. doi:10.1161/01.STR.0000166194.82027.63

7. Blanc R, Maia Barros AD, Brugieres P, Méder JF, Gaston A (2004) Cavernous sinus dural arteriovenous fistula complicated by edematous cerebral lesions from venous etiology. J Neuroradiol 31:220-224

8. Suzuki Y, Matsumoto K (2000) Variations of the superficial middle cerebral vein: classification using three-dimensional CT angiography. AJNR Am J Neuroradiol 21:932-938

9. Tanoue S, Kiyosue H, Okahara M, Sagara Y, Hori Y, Kashiwagi J, Mori H (2006) Para-cavernous sinus venous structures: anatomic variations and pathologic conditions evaluated on fat-suppressed 3D fast gradient-echo MR images. AJNR Am J Neuroradiol 27:10831089

10. Huang YP, Wolf BS, Antin SP, Okudera T (1996) The veins of the posterior fossa - anterior or petrosal draining group. Am J Roentgenol Radium Ther Nucl Med 104:36-56
11. Padget DH (1956) The cranial venous system in man in reference to development, adult configuration, and relation to the arteries. Am J Anat 98:307-355. doi:10.1002/aja.1000980302

12. Hashimoto M, Yokota A, Yamada H, Okudera T (2000) Development of cavernous sinus in the fetal period: a morphological study. Neurol Med Chir (Tokyo) 40:140-150. doi:10.2176/ nmc. 40.140

13. Knosp E, Müller G, Perneczky A et al (1987) Anatomical remarks on the fetal cavernous sinus and on the veins of the middle cranial fossa. In: Dolenc VV (ed) The cavernous sinus: a multidisciplinary approach to vascular and tumorous lesions. Springer-Verlag, New York, pp 104-116

14. Kazumata K, Kamiyama H, Ishikawa T et al (2003) Operative anatomy and classification of the sylvian veins for the transsylvian approach. Neurol Med Chir (Tokyo) 43:427-434. doi:10.2176/ nmc. 43.427

15. Kiyosue H, Hori Y, Okahara M et al (2004) Treatment of intracranial dural arteriovenous fistulas: current strategies based on location and hemodynamics, and alternative techniques of transcatheter embolization. Radiographics 24:1637-1653. doi:10.1148/ rg. 246045026

16. Matsushima T, Rhoton AL, Oliveira F, Peace D (1983) Microsurgical anatomy of the vein of the posterior fossa. J Neurosurg 59:63-105

17. Kim DJ, Kim DI, Shu SH et al (2006) Results of transvenous embolization of cavernous dural arteriovenous fistula: a singlecenter experience with emphasis on complications and management. AJNR Am J Neuroradiol 27:2078-2082 